ISSN 1855-3966 (printed edn.), ISSN 1855-3974 (electronic edn.)

\author{
ARS MATHEMATICA CONTEMPORANEA 16 (2019) 111-117 \\ https://doi.org/10.26493/1855-3974.1317.745 \\ (Also available at http://amc-journal.eu)
}

\title{
Weight choosability of oriented hypergraphs
}

\author{
Marcin Anholcer* \\ Faculty of Informatics and Electronic Economy, Poznan University of Economics and \\ Business, al. Niepodległości 10, 61-875 Poznań, Poland
}

\section{Bartłomiej Bosek}

Theoretical Computer Science Department, Faculty of Mathematics and Computer Science, Jagiellonian University, ul. Prof. S. Łojasiewicza 6, 30-348 Kraków, Poland

\section{Jarosław Grytczuk ${ }^{\dagger}$}

Faculty of Mathematics and Information Science, Warsaw University of Technology, ul. Koszykowa 75, 00-662 Warsaw, Poland

Received 4 February 2017, accepted 22 May 2018, published online 19 September 2018

\begin{abstract}
The 1-2-3 conjecture states that every simple graph (with no isolated edges) has an edge weigthing by numbers $1,2,3$ such that the resulting weighted vertex degrees form a proper coloring of the graph. We study a similar problem for oriented hypergraphs. We prove that every oriented hypergraph has an edge weighting satisfying a similar condition, even if the weights are to be chosen from arbitrary lists of size two. The proof is based on the Combinatorial Nullstellensatz and a theorem of Schur for permanents of positive semi-definite matrices. We derive several consequences of the main result for uniform hypergraphs. We also point on possible applications of our results to problems of 1-2-3 type for non-oriented hypergraphs.
\end{abstract}

Keywords: Oriented hypergraphs, 1-2-3 conjecture, combinatorial nullstellensatz, list weighting.

Math. Subj. Class.: 05C15, 05C65, 05C78

\footnotetext{
* Corresponding author.

${ }^{\dagger}$ Supported by the Polish National Science Center, decision nr DEC-2012/05/B/ST1/00652.

E-mail addresses: m.anholcer@ue.poznan.pl (Marcin Anholcer), bosek@tcs.uj.edu.pl (Bartłomiej Bosek), j.grytczuk@mini.pw.edu.pl (Jarosław Grytczuk)
}

() (i) This work is licensed under https://creativecommons.org/licenses/by/4.0/ 


\section{Introduction}

The famous 1-2-3 conjecture, posed by Karoński, Łuczak, and Thomason [8], states that every simple graph (with no isolated edges) has an edge weighting with integers $1,2,3$ such that no two adjacent vertices get the same weighted degrees. This innocently looking problem remains open for more than ten years, despite serious attacks based on various techniques, including the celebrated Combinatorial Nullstellensatz of Alon [1] (see [2, 10, 12]). The best result up to now [6] confirms the conjecture when the set of allowable weights includes also 4 and 5 . There were also many variants considered involving lists, orientations, and most recently hypergraphs (see [2, 3, 5, 7]). It is known, for instance, that any oriented graph has a weighting with numbers 1 and 2 only, such that the resulting weighted degrees are different for every pair of adjacent vertices [2, 9].

In this paper we consider the list version of the 1-2-3 conjecture for oriented hypergraphs. A hypergraph $H$ on the set of vertices $V$ is a family $E$ of non-empty subsets of $V$, called the edges of $H$. A hypergraph $H$ is $k$-uniform if each of its edges has size exactly $k$. So, a 2-uniform hypergraph is just a simple graph. Let $I_{H}$ denote the incidence graph of a hypergraph $H$, that is, a bipartite graph with color classes $V$ and $E$, whose edges are of the form $v e$, with $v \in V, e \in E$, whenever $v \in e$.

Now, by an orientation of a hypergraph $H$ we mean any function $\mu: E\left(I_{H}\right) \rightarrow \mathbb{C}$ assigning non-zero complex "signs" to the edges of the graph $I_{H}$. The cumulated degree of a vertex $v \in V$ is defined by

$$
D_{v}=\sum_{e \ni v} \mu(e v)
$$

If the range of the mapping $\mu$ is confined to the set $\{-1,+1\}$, or more generally, to the set of complex roots of unity, then we get a natural generalization of traditional orientation of a simple graph. The orientation $\mu$ is conveniently represented by the oriented incidence matrix $X=\left[\mu_{e v}\right]$ of dimension $|E| \times|V|$, where $\mu_{e v}=\mu(e v)$ if $e v \in E\left(I_{H}\right)$, and $\mu_{e v}=0$, otherwise. By an oriented hypergraph we mean a hypergraph $H$ together with some fixed orientation $\mu$.

Suppose now that each edge $e \in E$ of an oriented hypergraph $(H, \mu)$ is assigned a complex weight $w_{e}$. Then the resulting weighted degree of a vertex $v \in V$ is computed as

$$
W_{v}=\sum_{e \ni v} \mu_{e v} w_{e}
$$

For each $e \in E$ we now define

$$
W_{e}=\sum_{v \in e} \mu_{e v}^{*} W_{v},
$$

where $x^{*}$ denotes the complex conjugate of $x$. Observe that in case of a usual oriented graph, $W_{e}$ is exactly the difference between weighted degrees of both ends of $e$. We say that the weighting $w$ is virtuous if for each edge $e \in E$ we have

$$
W_{e} \neq 0
$$

Suppose that a list of complex numbers $L_{e}$ is assigned to each $e \in E$. We say that an oriented hypergraph $H$ is $t$-weight choosable if for any lists satisfying $\left|L_{e}\right| \geq t$ we are able to choose weights $w_{e} \in L_{e}$ so that $w$ is a virtuous weighting of $H$. Our main theorem extends the results of [2] and [9] in the following way. 
Theorem 1.1. Every oriented hypergraph is 2-weight choosable.

In the next section we will give an algebraic proof of this theorem based on the Combinatorial Nullstellensatz. It is inspired by the approach applied in [9]. In the last section we will speculate on possible consequences of this result for non-oriented hypergraphs, in particular, to the recent intriguing conjecture of Karoński, Kalkowski, and Pfender [7].

\section{Proof of the main result}

First recall the celebrated Combinatorial Nullstellensatz of Alon [1].

Theorem 2.1 (Alon). Let $K$ be an arbitrary field, and let $F=F\left(x_{1}, x_{2}, \ldots, x_{n}\right)$ be a polynomial in $K\left[x_{1}, x_{2}, \ldots, x_{n}\right]$. Suppose that the total degree of $f$ is $\sum_{i=1}^{n} t_{i}$, where each $t_{i}$ is a nonnegative integer, and suppose that the coefficient of $\prod_{i=1}^{n} x_{i}^{t_{i}}$ is nonzero. If $S_{1}, S_{2}, \ldots, S_{n}$ are subsets of $K$, with $\left|S_{i}\right|>t_{i}$, then there are $s_{1} \in S_{1}, s_{2} \in S_{2}, \ldots$, $s_{n} \in S_{n}$ so that

$$
F\left(s_{1}, s_{2}, \ldots, s_{n}\right) \neq 0
$$

Let $A=\left(a_{i j}\right)_{n \times n}$ be a square matrix with complex entries. Recall that the permanent of $A$ is defined by:

$$
\operatorname{per}(A)=\sum_{\sigma \in S_{n}} \prod_{i=1}^{n} a_{i, \sigma(i)},
$$

where $S_{n}$ denotes the group of permutations of the set $\{1,2, \ldots, n\}$. This is seemingly almost the same as the determinant of $A$, only the signs of permutations $\sigma$ are ignored. The following classic result of Schur [11] gives a relation between permanent and determinant of a Hermitian matrix.

Theorem 2.2 (Schur). If $A$ is a positive semi-definite Hermitian matrix, then $\operatorname{per}(A) \geq$ $\operatorname{det}(A)$, with equality if and only if $A$ is diagonal or $A$ has a zero row.

Proof of Theorem 1.1. Let $H$ be a hypergraph with $n$ vertices, $m$ edges, and fixed orientation $\mu$. Assume that a list $L_{e}$ with two complex numbers has been assigned to each edge $e \in E$ of $H$, as well as a complex variable $x_{e}$. Let us define

$$
P_{v}=\sum_{e \ni v} \mu_{e v} x_{e}
$$

for each vertex $v \in V$ of $H$, and

$$
P_{e}=\sum_{v \in e} \mu_{e v}^{*} P_{v}
$$

for each $e \in E$. Finally, let us define the complex multivariate polynomial

$$
P_{H}=\prod_{e \in E} P_{e} .
$$

We see that $H$ is 2 -weight choosable if

$$
P_{H}\left(w_{e_{1}}, w_{e_{2}}, \ldots, w_{e_{m}}\right) \neq 0
$$


for some choice of weights $w_{e} \in L_{e}$, with $e \in E$.

Each monomial in $P_{H}$ has total degree $m$. We are going to show that the coefficient of

$$
\prod_{e \in E} x_{e}
$$

is nonzero. This will finish the proof by Theorem 2.1.

Let us expand $P_{H}$. We have:

$$
P_{e}=\sum_{v \in e} \mu_{e v}^{*} P_{v}=\sum_{v \in e} \mu_{e v}^{*} \sum_{f \ni v} \mu_{f v} x_{f}=\sum_{v \in e} \mu_{e v}^{*} \sum_{f \in E} \mu_{f v} x_{f} .
$$

The sums in the last expression are independent, thus we can write

$$
P_{e}=\sum_{v \in e} \sum_{f \in E} \mu_{e v}^{*} \mu_{f v} x_{f}=\sum_{f \in E} \sum_{v \in e} \mu_{e v}^{*} \mu_{f v} x_{f} .
$$

Let

$$
m_{e f}=\sum_{v \in e} \mu_{e v}^{*} \mu_{f v} .
$$

Let $M$ be the $m \times m$ matrix consisting of the $m_{e f}$. From the above definition it follows that $M=X X^{*}$, where $X$ is the oriented incidence matrix of $H$, and $X^{*}$ is its conjugate transpose.

We have

$$
P_{e}=\sum_{f \in E} m_{e f} x_{f},
$$

and

$$
P_{H}=\prod_{e \in E} P_{e}=\prod_{e \in E} \sum_{f \in E} m_{e f} x_{f} .
$$

It follows that the coefficient of

$$
\prod_{e \in E} x_{e}
$$

is equal to $\operatorname{per}(M)$. So, it is enough to prove that $\operatorname{per}(M) \neq 0$ (see the permanent lemma in [1]). To get this we will apply Schur's theorem. First notice that for any complex vector $z$ we have

$$
z M z^{*}=z X X^{*} z^{*}=z X(z X)^{*}=|z X|^{2} .
$$

As the last number is real and non-negative, $M$ is positive semi-definite. Notice also that by assumption $H$ has no empty edges and $\mu_{e v} \neq 0$ for all $v \in e$. Therefore all entries on the main diagonal of $M$ are strictly positive real numbers. In particular, $M$ has no zero row. Hence, if $M$ is not diagonal, then by Schur's theorem we get

$$
\operatorname{per}(M)>\operatorname{det}(M) \geq 0 .
$$

If $M$ is diagonal, then

$$
\operatorname{per}(M)=\operatorname{det}(M)=\prod_{e \in E} m_{e e}>0 .
$$

This completes the proof. 


\section{Some applications for uniform hypergraphs}

We give two applications of Theorem 1.1 extending some results from [2, 5] and [9]. For simplicity we confine ourselves to uniform hypergraphs with specific orientations defined as follows. Let $k \geq 2$ be a fixed positive integer and let $U_{k}$ denote the multiplicative group of $k$-th complex roots of unity. If $\varepsilon$ is a primitive root in $U_{k}$, then we may write $U_{k}=\left\{1, \varepsilon, \varepsilon^{2}, \ldots, \varepsilon^{k-1}\right\}$.

Let $H$ be a $k$-uniform hypergraph. Consider a canonical orientation of $H$ given by the mapping $\mu: E\left(I_{H}\right) \rightarrow U_{k}$ such that $\mu(e u) \neq \mu(e v)$ for every edge $e \in E$ and any two vertices $u, v \in e$. Notice that for $k=2$ we get the traditional orientation of a simple graph. Recall that a coloring of the vertices of a hypergraph is proper if no edge is monochromatic.

Corollary 3.1. Every canonically oriented $k$-uniform hypergraph $H$ has an edge weighting with numbers 1, 2 such that weighted vertex degrees give a proper coloring of $H$.

Proof. Let $H$ be a given $k$-uniform hypergraph and let $\mu$ be any canonical orientation of $H$. Assign the lists $L_{e}=\{1,2\}$ to all edges of $H$. Then by Theorem 1.1 there exists a virtuous edge weighting $w$ such that $w_{e} \in\{1,2\}$ for every $e \in E$. We claim that this weighting satisfies the assertion of the corollary. Suppose on the contrary that this is not the case, and that there is some edge $e=\left\{v_{1}, v_{2}, \ldots, v_{k}\right\}$ such that all weighted degrees $W_{v_{i}}$ are equal:

$$
W_{v_{1}}=\cdots=W_{v_{k}}=W
$$

This implies that

$$
W_{e}=\sum_{i=1}^{k} \mu\left(e v_{i}\right)^{*} W_{v_{i}}=W \sum_{i=1}^{k} \mu\left(e v_{i}\right)^{*}=0
$$

since

$$
\sum_{i=1}^{k} \mu\left(e v_{i}\right)^{*}=1+\varepsilon+\cdots+\varepsilon^{k-1}=0 .
$$

This contradicts the virtue of the weighting $w$.

Corollary 3.2. Every $k$-uniform hypergraph $H$ has a canonical orientation such that cumulated vertex degrees give a proper coloring of $H$.

Proof. Let $H$ be a given $k$-uniform hypergraph and let $\mu$ be any of its canonical orientations. Assign the list $L_{e}=\{1, \varepsilon\}$ to every edge $e \in E$. Then by Theorem 1.1 there exists a virtuous edge weighting $w$ such that $w_{e} \in\{1, \varepsilon\}$ for every $e \in E$. Consider now a new orientation $\mu^{\prime}$ defined by $\mu^{\prime}(e v)=\mu(e v) w_{e}$ for every edge $e v$ of the incidence graph $I_{H}$. We claim that this orientation satisfies the assertion of the corollary. Suppose on the contrary that this is not the case, and that there is some edge $e=\left\{v_{1}, v_{2}, \ldots, v_{k}\right\}$ with all cumulated degrees $D_{v_{i}}$ equal in orientation $\mu^{\prime}$ :

$$
D_{v_{1}}=\cdots=D_{v_{k}}=D
$$

This implies that also weighted degrees $W_{v_{i}}$ are equal to $D$, since

$$
W_{v_{i}}=\sum_{e \ni v_{i}} \mu\left(e v_{i}\right) w_{e}=\sum_{e \ni v_{i}} \mu^{\prime}\left(e v_{i}\right)=D_{v_{i}}=D \text {. }
$$


In consequence, we get

$$
W_{e}=\sum_{i=1}^{k} \mu^{*}\left(e v_{i}\right) W_{v_{i}}=D \sum_{i=1}^{k} \mu\left(e v_{i}\right)^{*}=0
$$

which contradicts the fact that $w$ is virtuous.

\section{Discussion}

We conclude the paper with pointing on some possible applications of our results to nonoriented problems of "1-2-3" type. Actually, the original 1-2-3 conjecture can be stated in a setting similar to virtuous weightings of oriented hypergraphs. To see this consider a bipartite graph $B$ on bipartition classes $X$ and $Y$ with some signing $\mu: E(B) \rightarrow\{-1,+1\}$. Let $w: X \rightarrow \mathbb{Z}$ be a weighting of one part of $B$. Then for every vertex $y \in Y$ we may define the induced weight of $y$ by

$$
W_{y}=\sum_{x \in N(y)} w(x) \mu(x y)
$$

where $N(y)$ denote the set of neighbors of $y$. A weighting $w$ is called half-virtuous if $W_{y} \neq 0$ for every $y \in Y$. A natural problem is to find for a given signed graph $B$, the least integer $k$ such that $B$ has a half-virtuous weighting with $w(x) \in\{1,2, \ldots, k\}$ for each $x \in X$. It is not hard to see that 1-2-3-conjecture is equivalent to the statement that certain signed bipartite graphs arising from simple graphs have half-virtuous weightings with weights in the range $\{1,2,3\}$. Consider a simple graph $G$ and the related bipartite graph $B_{G}$ on bipartition classes $X=Y=E(G)$, with $x y \in E\left(B_{G}\right)$ whenever $x$ and $y$ are incident edges of $G$. An appropriate signing $\mu$ is defined so that for a fixed $y \in Y$, the edges $x y, x^{\prime} y \in E\left(B_{G}\right)$ have the same sign if and only if $x$ and $x^{\prime}$ are incident in $G$ to the same end of $y$. A half-virtuous weighting of $B_{G}$ is then equivalent to a weighting of the edges of $G$ with different sums over edges incident to opposite ends of any given edge.

It is also possible that our results could be useful in a recently introduced version of the 1-2-3 conjecture for (non-oriented) hypergraphs [7]. Let $H$ be a $k$-uniform hypergraph and let $w$ denote a weighting of its edges. For every vertex $v$ define its weighted degree by

$$
W_{v}=\sum_{e \ni v} w_{e}
$$

Recall that a proper coloring of $H$ is a coloring of its vertices such that no edge is monochromatic. A hypergraph $H$ is $r$-weight colorable if there is a weighting $w: E(H) \rightarrow\{1,2, \ldots$, $r\}$ such that the weighted degrees $W_{v}$ form a proper coloring of $H$. The following conjecture is stated in [4] (see also [7]).

Conjecture 4.1. Every $k$-uniform hypergraph $(k \geq 2)$ with no isolated edges is 3-weight colorable.

The conjecture holds for random uniform hypergraphs in a stronger sense that even non-weighted degrees give a proper coloring, as proved recently in [4]. Another strong support for validity of the conjecture is given in [7], where it is proved that every nontrivial hypergraph (not only uniform) is $(2,3)$-weight colorable. This means that a proper 
coloring of a hypergraph is obtained by using weights $\{1,2,3\}$ for the edges, and weights $\{1,2\}$ for the vertices, with weighted vertex degrees computed by a formula:

$$
W_{v}=w_{v}+\sum_{e \ni v} w_{e}
$$

This statement in restriction to simple graphs was formerly proved by Kalkowski in his $\mathrm{PhD}$ thesis. Then the result was extended to the list version by Wong and Zhu [12] who applied the Combinatorial Nullstellensatz. This encourages us to conclude the paper with the following general conjecture.

Conjecture 4.2. Every $k$-uniform hypergraph $(k \geq 2)$ with no isolated edges is 3-weight choosable.

\section{References}

[1] N. Alon, Combinatorial Nullstellensatz, Combin. Probab. Comput. 8 (1999), 7-29, doi:10. 1017/s0963548398003411.

[2] T. Bartnicki, J. Grytczuk and S. Niwczyk, Weight choosability of graphs, J. Graph Theory 60 (2009), 242-256, doi:10.1002/jgt.20354.

[3] O. Baudon, J. Bensmail and É. Sopena, An oriented version of the 1-2-3 conjecture, Discuss. Math. Graph Theory 35 (2015), 141-156, doi:10.7151/dmgt.1791.

[4] P. Bennett, A. Dudek, A. Frieze and L. Helenius, Weak and strong versions of the 1-2-3 conjecture for uniform hypergraphs, Electron. J. Combin. 23 (2016), \#P2.46, http://www . combinatorics.org/ojs/index.php/eljc/article/view/v23i2p46.

[5] M. Borowiecki, J. Grytczuk and M. Pilśniak, Coloring chip configurations on graphs and digraphs, Inform. Process. Lett. 112 (2012), 1-4, doi:10.1016/j.ipl.2011.09.011.

[6] M. Kalkowski, M. Karoński and F. Pfender, Vertex-coloring edge-weightings: towards the 12-3-conjecture, J. Comb. Theory Ser. B 100 (2010), 347-349, doi:10.1016/j.jctb.2009.06.002.

[7] M. Kalkowski, M. Karoński and F. Pfender, The 1-2-3-conjecture for hypergraphs, J. Graph Theory 85 (2017), 706-715, doi:10.1002/jgt.22100.

[8] M. Karoński, T. Łuczak and A. Thomason, Edge weights and vertex colours, J. Comb. Theory Ser. B 91 (2004), 151-157, doi:10.1016/j.jctb.2003.12.001.

[9] M. Khatirinejad, R. Naserasr, M. Newman, B. Seamone and B. Stevens, Digraphs are 2-weight choosable, Electron. J. Combin. 18 (2011), \#P21, http: / / www. combinatorics.org/ ojs/index.php/eljc/article/view/v18ilp21.

[10] J. Przybyło and M. Woźniak, Total weight choosability of graphs, Electron. J. Combin. 18 (2011), \#P112, http://www.combinatorics.org/ojs/index.php/eljc/ article/view/v18ilp112.

[11] I. Schur, Über endliche Gruppen und Hermitesche Formen, Math. Z. 1 (1918), 184-207, doi: 10.1007/bf01203611.

[12] T.-L. Wong and X. Zhu, Every graph is (2,3)-choosable, Combinatorica 36 (2016), 121-127, doi:10.1007/s00493-014-3057-8. 